\section{FIRST PHASE 2 RESULTS OF AUTOLOGOUS TUMOR- INFILTRATING LYMPHOCYTE (TIL; LN-145) MONOTHERAPY IN PATIENTS WITH ADVANCED, IMMUNE CHECKPOINT INHIBITOR-TREATED, NON- SMALL CELL LUNG CANCER (NSCLC)}

\begin{abstract}
${ }^{1}$ Adam Schoenfeld*, ${ }^{2}$ Sylvia Lee, ${ }^{3}$ Luis Paz-Ares, ${ }^{4}$ Bernard Doger, ${ }^{5}$ Scott Gettinger, ${ }^{6}$ Simon Haefliger, ${ }^{7}$ Angela Orcurto, ${ }^{8}$ Ammar Sukari, ${ }^{9}$ Sophie Papa, ${ }^{10}$ Juan Francisco Rodriguez Moreno, ${ }^{11}$ Friedrich Graf Finckenstein, ${ }^{11}$ Madan Jagasia, ${ }^{11}$ Rana Fiaz, ${ }^{11}$ Giri Sulur, ${ }^{11}$ Guang Chen, ${ }^{11}$ Viktoria Gontcharova, ${ }^{12}$ Kai He. ${ }^{1}$ Memorial Sloan Kettering Cancer Center, New York, NY, USA; ${ }^{2}$ Fred Hutchinson Cancer Research Center, Seattle, WA, USA; ${ }^{3}$ Hospital Universitario 12 de Octubre, Madrid, Spain; ${ }^{4}$ Hospital Universitario Fundacion Jimenez Diaz, Madrid, Spain; ${ }^{5}$ Yale Cancer Center, New Haven, CT, USA; ${ }^{6}$ Universitätsspital Bern, Bern, Switzerland; ${ }^{7}$ Centre Hospitalier Universitaire Vaudois, Lausanne, Switzerland; ${ }^{8}$ Karmanos Cancer Institute, Detroit, MI, USA; ${ }^{9}$ Guy's and St Thomas' NHS Foundation Trust, London, UK; ${ }^{10}$ Hospital Universitario HM Sanchinarro, Madrid, Spain; "11 lovance Biotherapeutics, Inc., San Carlos, CA, USA; ${ }^{12}$ Ohio State University, Columbus, OH, USA
\end{abstract}

Background A majority of patients with advanced NSCLC develop disease progression with first-line immune-checkpoint inhibitors (ICI) \pm chemotherapy. In the setting of ICI resistance, effective strategies to provide deep and durable responses are urgently needed. Lifileucel (LN-144) and LN145 are centrally manufactured (cryopreserved drug-product, 22-day manufacturing process) autologous TIL products that have demonstrated activity in advanced melanoma, cervical cancer, and head and neck carcinoma. ${ }^{1-4}$ Here, we report the first safety and efficacy data for LN-145 as monotherapy in patients with advanced NSCLC.

Methods IOV-COM-202 (NCT03645928) is a phase 2 multicenter, multicohort, open-label study evaluating autologous TIL cell therapy in patients with solid tumors. We report data from Cohort 3B, investigating LN-145 monotherapy in patients with advanced or metastatic NSCLC. Eligibility required 1-3 prior lines of systemic therapy, including either ICI or oncogene-directed therapy. Treatment included nonmyeloablative lymphodepletion, TIL infusion, and $\leq 6$ interleukin-2 doses. Primary endpoints were safety (incidence of Grade $\geq 3$ treatment-emergent adverse events [TEAEs]) and objective response rate (ORR, investigator-assessed using RECIST v1.1). Exploratory biomarker analyses, including T-cell receptor (TCR) repertoire, were performed.

Results As of 24June2021, 28 patients received LN-145 (fullanalysis set [FAS]; table 1) and 24 were efficacy-evaluable; all had received prior ICI. TIL were most commonly harvested from lung metastases $(57.1 \%)$. Safety was consistent with the underlying disease and known TEAE profiles of nonmyeloablative lymphodepletion and interleukin-2. Grade $\geq 3$ TEAEs in $\geq 30 \%$ of patients were thrombocytopenia and anemia. The ORR in the FAS and efficacy-evaluable set was $21.4 \%(6 / 28)$ and 25.0\% (6/24; figure 1), respectively. Median duration of response was not reached and $83 \%(5 / 6)$ of responses were ongoing at last follow-up (median study follow-up, 8.2 months). One patient had a complete metabolic response, ongoing at 20.7 months; 2 responses occurred in patients who were PD-L1-negative. All responders received $\geq 2$ prior lines of systemic therapy. Twenty-six patients had TIL available from the final drug-product for TCR repertoire analysis; mean (min-max) number of unique TCR clones was 13,142 (3093$35,734)$ and Shannon Entropy index was 7.34 (3.7-12). Updated data will be presented.
Best Percentage Change from Baseline in Target Lesion Sum of Diameters for Efficacy-Evaluable Set

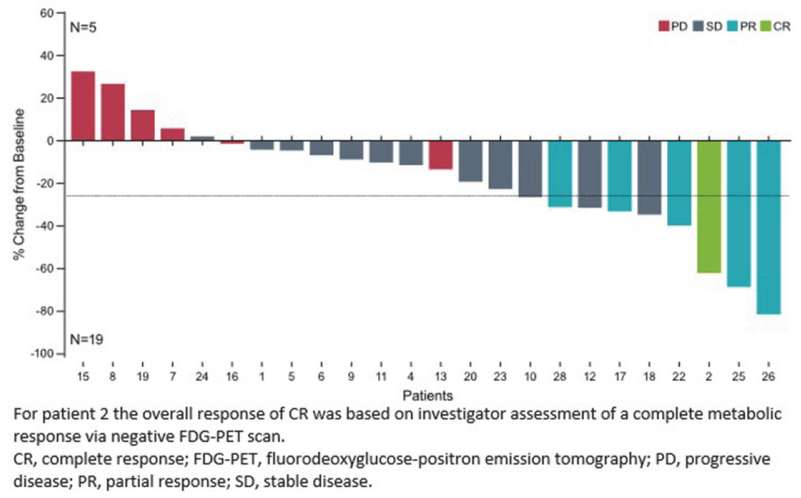

Abstract 458 Figure 1 Best percentage change from baseline in target lesion sum of diameters for efficacy-evaluable set

Abstract 458 Table 1 Baseline patient demographic and clinical characteristics; efficacy parameters

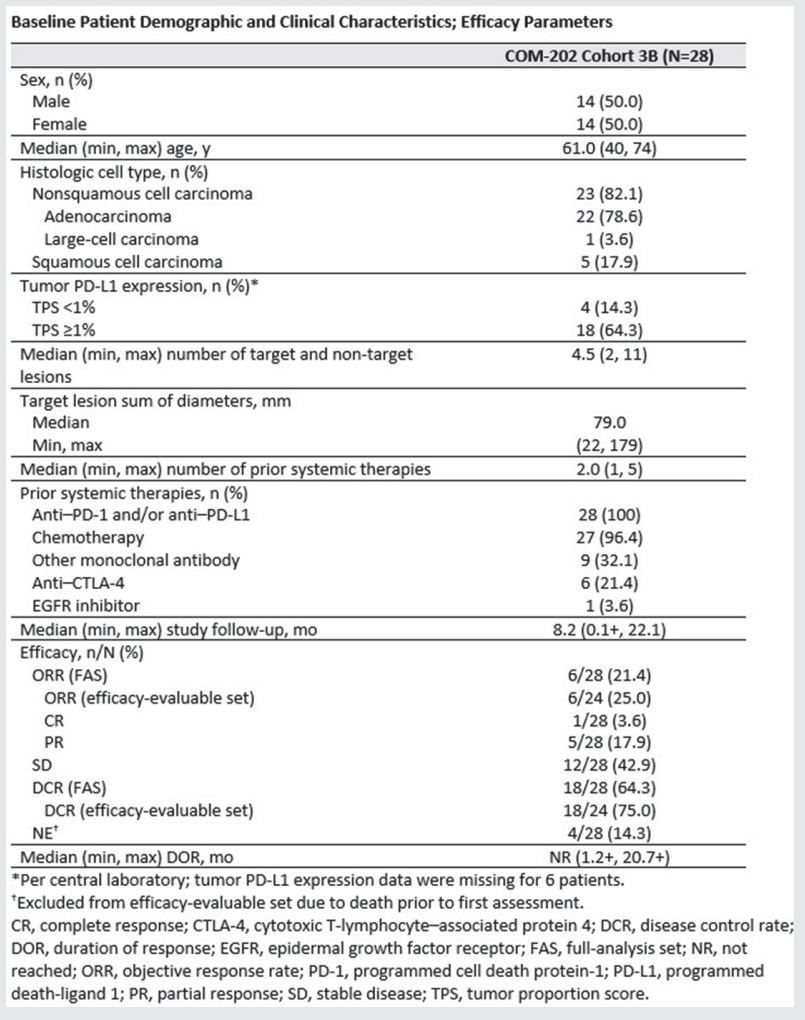

Conclusions LN-145 was successfully manufactured and onetime treatment produced an expected safety profile and durable responses in heavily pretreated patients with NSCLC, regardless of PD-L1 expression. The activity of LN-145 monotherapy is encouraging and warrants further investigation of LN-145 as a single-agent and in combination in patients with NSCLC in ongoing studies IOV-LUN-202 (NCT04614103) and IOV-COM-202 Cohorts 3A and 3C (3B closed to enrollment).

Acknowledgements This study and analysis were funded by Iovance Biotherapeutics, Inc. (San Carlos, CA, USA). Writing support was provided by Amanda Kelly (Iovance); graphics support was provided by Cognition Studio (Seattle, WA, USA). Trial Registration NCT03645928 


\section{REFERENCES}

1. Sarnaik AA, et al. J Clin Oncol 2021; doi: 10.1200/JC0.21.00612.

2. Thomas SS, et al. J Clin Oncol 2021;39: (suppl; abstract 9537).

3. Jazaeri A, et al. J Clin Oncol 2019;37: (suppl; abstract 2538).

4. Jimeno A, et al. J Immunother Cancer 2020;8: (suppl; abstract A378).

Ethics Approval The study was approved by Advarra Institutional Review Board, approval number Pro00035064 and all study participants provided written consent via signature of the IRB-approved Informed Consent form.

http://dx.doi.org/10.1136/jitc-2021-SITC2021.458 